\title{
Letter
}

\section{The Composition and Evolutionary Status of Proxima Centauri}

\author{
Martin Beech ${ }^{1,2}$, Corey McCowan ${ }^{1}$, Lowell Peltier ${ }^{2}$ \\ ${ }^{1}$ Campion College, The University of Regina, SK, Canada \\ ${ }^{2}$ Department of Physics, The University of Regina, SK, Canada
}

Email address:

beechm@uregina.ca (M. Beech), corey.mccowan@outlook.com (C. McCowan), peltie21@uregina.ca (L. Peltier)

\section{To cite this article:}

Martin Beech, Corey McCowan, Lowell Peltier. The Composition and Evolutionary Status of Proxima Centauri. American Journal of Astronomy and Astrophysics. Vol. 5, No. 1, 2017, pp. 1-5. doi: 10.11648/j.ajaa.20170501.11

Received: January 7, 2017; Accepted: January 21, 2017; Published: February 22, 2017

\begin{abstract}
A suite of stellar evolution models has been used to estimate the mass and metallicity of Proxima Centauri (GJ 551, HIP 70890, V645 Cen). It is found that the observations are best described by an $M \approx 0.12 \mathrm{M}_{\odot}$ star with a heavy element mass fraction in the range $0.004<\mathrm{Z}<0.01$ (or equivalently, a metallicity of $-0.5<[\mathrm{Fe} / \mathrm{H}]<-0.3$ ). The derived metallicity of Proxima is distinctly at odds with that established for $\alpha$ Cen A and $\alpha$ Cen B. It is argued that both the observational data as well as the evolutionary models for Proxima Centauri are consistent with an age of some 7 to 8 Gyr and that its (presently derived) physical characteristics are inconsistent with an in situ or coevally origin with the $\alpha$ Cen AB binary.
\end{abstract}

Keywords: Proxima Centauri, $\alpha$ Cen A, $\alpha$ Cen B, Stellar Evolution, Metallicity

\section{Introduction}

Proxima Centauri is the closest star to the solar system at the present epoch. Discovered by Robert Innes in 1915, it was soon realized that while Proxima was located some 2.2 degrees away from alpha-Centauri on the sky it, none-the-less, had a common proper motion with that system [1]. As such, Proxima, while being physically separated from alpha-Centauri by $\sim 13,000 \mathrm{AU}$ at the present time, has long been taken as a distant third companion (alpha-Centauri $\mathrm{C}$ ) to the $\alpha$ Cen AB binary. Doubts concerning the third member companionship of Proxima to $\alpha$ Cen $\mathrm{AB}$ have been raised on a number of occasions and it seems clear that, at best, Proxima is only marginally bound under standard Newtonian gravitational conditions [2, 3, 4], and that under alternative scenarios $[5,6]$ it fits well within the domain of Modified Newtonian Dynamics (MOND). Kervella, Thevenin \& Lovis [7] have most recently investigated the orbital dynamics of Proxima, and while they conclude that it is a gravitationally bound object to $\alpha$ Cen $\mathrm{AB}$, their analysis, while claiming a "high degree of confidence" is still not fully convincing - indeed, they find, as with other earlier studies, that the odds of Proxima being a gravitationally bound object to $\alpha$ $\mathrm{Cen} \mathrm{AB}$ are not much better than 50:50. Indeed, the conclusions drawn by Kervella, Thevenin and Lovis are all in the interpretation of the observational uncertainties, and in the modeling of complicated spectral effects such as extensive line blending, convective blueshift and gravitational redshift. At the present time, and in the context of Scottish jurisprudence, the bound orbital status of Proxima Centauri to $\alpha$ Cen $\mathrm{AB}$ remains 'un-proven', and accordingly we look for alternative methods that might cement or negate its third companion (that is alpha-Centauri C) status. One such alternative method is to look at the observed physical characteristics of Proxima and attempt to assess their consistency with expectation under the assumption of a coeval origin with $\alpha$ Cen $\mathrm{AB}$. Various studies indicate that $\alpha$ Cen $\mathrm{A}$ and $\alpha$ Cen $\mathrm{B}$ have comparable ages and compositions $[8,9,10]$ - as would be expected for the components of a relatively close binary system. Indeed, the current consensus suggests an age of $5.0 \pm 0.5$ Gyr for the $\alpha$ Cen $\mathrm{AB}$ system, with each star having an elevated metallicity 
of some 1.5 times solar - that is a metallicity of $[\mathrm{Fe} / \mathrm{H}] \sim 0.21$ and a corresponding heavy element mass fraction of $\mathrm{Z} \approx 0.03$. Given this information, we can ask the question whether the physical properties of Proxima, that is its observed radius, luminosity and temperature, are consistent with its deduced mass of $0.12 \mathrm{M}_{\odot}$, an age estimate of 5 billion years, and a heavy element mass fraction of $Z=0.03$.

\section{Model Calculations}

The relative closeness of Proxima Centauri to the Sun has allowed for the derivation, by entirely independent observational methods, of well-defined values for its mass, radius, luminosity and temperature (table 1). Indeed, the mass, radius and temperature are known to within an uncertainty of about $5 \%$ - its luminosity is a little less well constrained, but measured to an uncertainty of about $10 \%$. Two values of the radius estimate are given in Table 1: the first value (INT) is that derived interferometrically by Demory et al. [11], while the second (ML) is that given by Kervella, Thevenin \& Lovis [7] based upon the recent $2 \mathrm{MASS} K_{s}$ magnitude-radius calibration given by Mann et al [12]. In terms of fitting stellar evolutionary models to the observed data, Proxima is particularly interesting in that the only unknown quantity is its composition, and specifically its metallicity. The Vogt-Russell theorem (see e.g., references [13, 14]), however, asserts that once the mass and composition of a star are set, so the equations of stellar structure must yield unique expressions for the radius, temperature and luminosity, and accordingly we argue that the physical properties of Proxima are sufficiently well constrained that an estimate of its underlying metallicity can be gauged via the tabulated results of numerical stellar models. If the metallicity of Proxima is consistent with that derived for $\alpha$ Cen $\mathrm{A}$ and $\mathrm{B}$, then a coeval origin is indicated - if, on the other hand, the compositional values are significantly different, then the implication is that Proxima did not form in the same place and/or at the same time as $\alpha$ Cen AB.

Table 1. The observational data pertaining to Proxima Centauri. INT and ML refer to the data points shown in figure 2.

\begin{tabular}{llll}
\hline Quantity & value & uncertainty & Ref. \\
\hline Mass $\left(\mathrm{M}_{\odot}\right)$ & 0.123 & \pm 0.006 & {$[11],[15]$} \\
Radius $\left(\mathrm{R}_{\odot}\right)$ & 0.141 & \pm 0.007 & {$[11](\mathrm{INT})$} \\
Luminosity $\left(\mathrm{L}_{\odot}\right)$ & 0.1543 & \pm 0.0045 & {$[7](\mathrm{ML})$} \\
Temperature $(\mathrm{K})$ & 0.0017 & \pm 0.0002 & {$[16]$} \\
\hline
\end{tabular}

In this analysis we have used the Ez-Web [17, 18] public domain stellar evolution code. This computer code has been developed around the Eggleton evolutionary model [19] with appropriately updated in-put physics. Figures 1 and 2 indicate the results from a series of calculations for stars having masses in the range $0.1<\mathrm{M} / \mathrm{M}_{\odot}<0.135$ - the mass range that encompasses that expected for Proxima. In figure 1, the solid diagonal line to the right in the diagram indicates the zero-age main sequence for stars having $Z=0.03$. Likewise, the upper solid diagonal line in figure 2 indicates the mass-radius relationship for stars with $\mathrm{Z}=$
0.03. It is immediately evident that neither the mass-radius relationship nor the luminosity-temperature characteristics, at any mass in our adopted range, with a $Z=0.03$ composition, are consistent with the observational data derived for Proxima. In order to bring the stellar models simultaneously closer to the derived values for the mass, radius, luminosity and temperature, a lower heavy element abundance is implicated. The two loci running to the upper left in figure 1 show a series of models, at an age of $1 \mathrm{Gyr}$, for stars having $\mathrm{M}=0.123 \mathrm{M}_{\odot}$ and $\mathrm{M}=0.117 \mathrm{M}_{\odot}$ with varying values for the heavy element mass fraction $Z$. Likewise the vertical (green circle) data points in figure 2 reveal the changes in radius at a fixed mass of $\mathrm{M}=0.123 \mathrm{M}_{\odot}$ but with selectively reduced values of the heavy element abundance. As expected from standard theory, the consequences of reducing the heavy element mass fraction, for a fixed total mass, is to decrease the radius but increase the temperature and luminosity [13, 14]. We find a good model match to the observed mass-radius (INT value in figure 2) and temperature-luminosity data for Proxima with a 1 Gyr old, $M=0.118 \mathrm{M}_{\odot}$ star having $\mathrm{Z}=0.004$ (or equivalently, $[\mathrm{Fe} / \mathrm{H}] \approx-0.5$ : see table 2 ). If, however, and there is no overriding reason to do so, preference is given to the mass and radius estimates provided by Kervella, Thevenin \& Lovis [7] (the ML data point in figure 2), then only a relatively poor model fit can be simultaneously found between the mass, radius, temperature and luminosity. The best model fit to the data in this latter case (with the temperature being least well accounted with $\Delta \mathrm{T}>100 \mathrm{~K})$ corresponds to that of a star of mass $0.12 \mathrm{M}_{\odot}$ and $\mathrm{Z}$ $=0.01($ or $[\mathrm{Fe} / \mathrm{H}] \approx-0.3)$.

Irrespective of which mass-radius data set is used (INT or ML in figure 2) it appears from standard stellar evolution model calculations that Proxima has a significantly lower metallicity than $\alpha$ Cen A or $\alpha$ Cen B. This result argues against Proxima being coeval with $\alpha$ Cen $\mathrm{AB}$, and accordingly it may not be appropriate to label it as alpha-Centauri $\mathrm{C}$

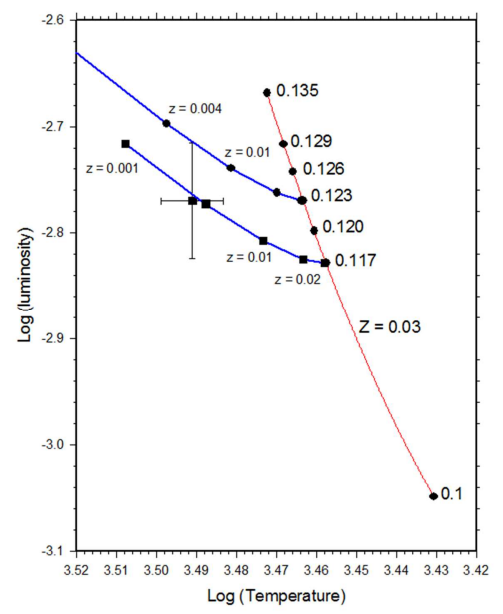

Figure 1. Hertzsprung-Russell diagram for 1 Gyr stellar models with various values of heavy element mass fraction $Z$. The main sequence corresponds to stars in the mass range $0.1<M / M_{\odot}<0.135$ (labelled data points) and with $Z$ $=0.03$. The data point with error bars indicates the uncertainty in the observed temperature and luminosity of Proxima. 


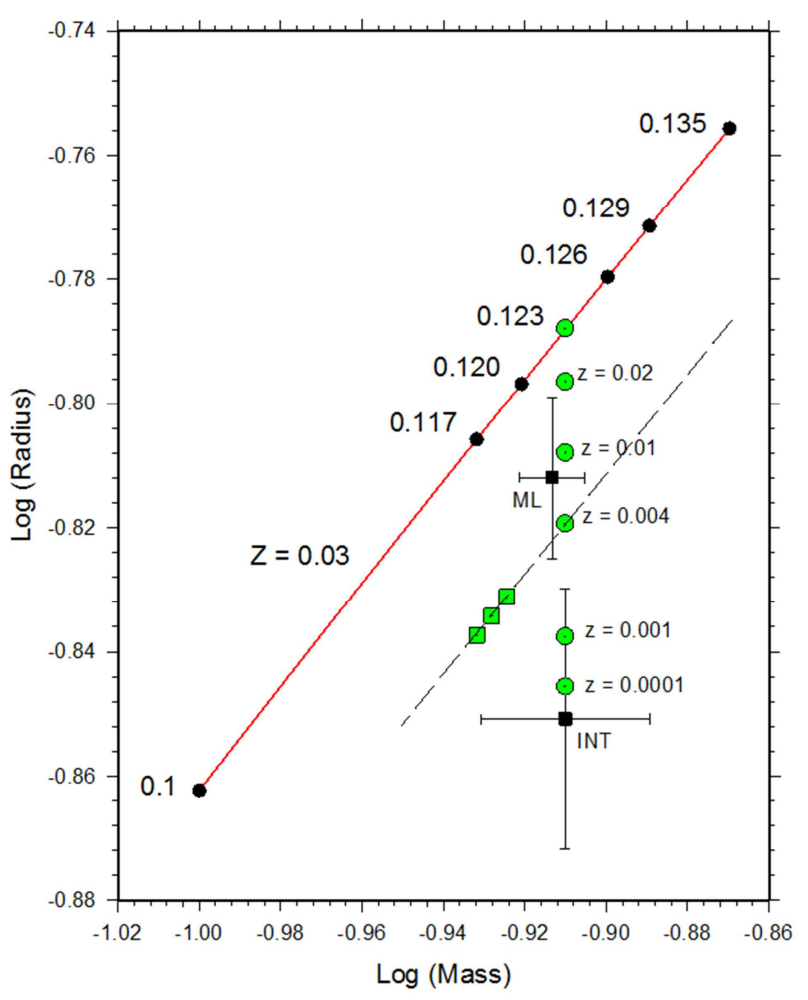

Figure 2. Mass-radius diagram for 1 Gyr stellar models with various values of the heavy element mass fraction $Z$. The data points with error bars indicate the uncertainty in the observed mass and radius of Proxima as derived by interferometry (INT) [11] and by the radius- $M_{K}$ relationship $(M L)$ [7, 12]. The solid (red) diagonal line corresponds to the mass-radius relationship for $Z=$ 0.03 and the data points are labelled according to stellar mass. The green dots 'displaced'downward at a fixed mass of 0.123 indicate the radius for various $0.0001 \leq Z \leq 0.02$ models. The three small, green-square, data points on the $Z$ $=0.004$ line correspond to 1 Gyr old stars having a mass of 0.117, 0.118 (see table 2) and $0.119 \mathrm{M}_{\odot}$

Table 2. Comparison between the 1 Gyr old, $M=0.118 M_{\odot}, Z=0.004$ model, and the observational data (see Table 1 for references to the observational data terms)

\begin{tabular}{lll}
\hline Mass $=\mathbf{0 . 1 1 8} \mathbf{M}_{\odot}$ & Model & Observations \\
\hline $\mathrm{Z}=0.004 \equiv[\mathrm{Fe} / \mathrm{H}] \approx-0.5 \mathrm{Age}=1 \mathrm{Gyr}$ & & \\
Luminosity $\left(\mathrm{L}_{\odot}\right)$ & $0.0017(4)$ & 0.0017 \\
Temperature $(\mathrm{K})$ & 3085 & 3098 \\
Radius $\left(\mathrm{R}_{\odot}\right)$ & 0.146 & 0.141 \\
\hline
\end{tabular}

\section{Discussion}

The complex atomic spectra exhibited by M-spectral type, dwarf stars have long proved a problem against the measure of their metallicity (see e.g., Schlaufman and Laughlin [20]). Indeed, the presence of strongly blended molecular lines, and the intrinsic faintness of $\mathrm{M}$ dwarfs in general, make the determination of the continuum a distinct challenge, and accordingly no detailed metallicity analysis has ever been published for Proxima Centauri. It is because of this presently insurmountable problem that a secondary method for determining the metallicity of Proxima has been investigated. Indeed, stellar models have long been used to estimate individual star masses, fundamental star characteristics and cluster ages, and the model-matching method has been highly successful in the case of $\alpha$ Cen A and $\alpha$ Cen B [8, 9]. One of the issues with respect to computing models for very low mass stars, however, is that of describing their atmospheres. This is important not only for setting the outer boundary conditions needed for solving the equations of stellar structure, but also for describing the photometric properties of such stars. Accordingly, as a test for consistency, the output (specifically radius, temperature and luminosity for a given mass) from the Ez-Web models have been compared against those published by Brocato, Cassisi and Castellani [21], Cassisi [22], and Baraffe et al. [23] over the same metallicity ranges. Encouragingly, the Ez-Web models are found to be in very good agreement with these earlier publications. This consistency between models (which incorporate different in-put physics and model atmospheres) is partly due to the fact that the stars in the mass range of interest here have fully convective interiors, and that the models of such stars are not sensitive to the otherwise uncertain values associated with any adopted mixing-length theory [24]. Additionally, in the mass range being considered, while degeneracy is beginning to become important, it is not excessive and/or predominant throughout the interior (in contrast to white dwarf models), and accordingly no specific accommodations are required with respect to applying a more complex equation of state.

The high age-degeneracy exhibited by the radius, luminosity and temperature variations of very low mass stars is such that the close model fit obtained at $1 \mathrm{Gyr}$ is also consistent with any age up to $\sim 10$ to $12 \mathrm{Gyr}$. Indeed, the low $-0.5<[\mathrm{Fe} / \mathrm{H}]<-0.3$ metallicity implicated for Proxima Centauri from the stellar evolutionary models is indicative of it potentially being an old-thin, or possibly thick-disk population object. Invoking an old-thin to thick-disk origin for Proxima means, however, that we are beginning to play against the odds with respect to its presently observed location and space velocity. The odds of Proxima being located at its present distance from $\alpha$ Cen $\mathrm{AB}$ are of order 1 in 57,000 [3,5]. On the other hand, Proxima's galactic $\mathrm{V}$ and $\mathrm{W}$ space velocity components are about that expected from the age-velocity dispersion relationship for old-thin and thick-disk stars - its U-velocity component, however, is about a factor of 10 smaller than might be expected for a thick disk member. Haywood [25] finds that about $10 \%$ of stars in the solar neighbourhood belong to the old-thin disk population, with $[\mathrm{Fe} / \mathrm{H}] \sim-0.3$, and about $5 \%$ of stars in the solar neighbourhood are derived from the thick-disk population, with $[\mathrm{Fe} / \mathrm{H}] \sim-0.5$. For all this, however, these low probability results, while not necessarily encouraging, may simply be the result of Proxima having had an unusual dynamical history [6].

In addition to its closeness to the solar system, Proxima has also been observed as an active flare star [26, 27], although how it (or, for that matter, any other M-spectral type star) generates and maintains a long-lived and coherent magnetic field is still not fully understood $[28,10]$. Using Sloan Digital Sky Survey data, however, West et al. [29] find that M5 V stars (i.e., ones having a spectral type similar to Proxima) have a 7.0 \pm 0.5 Gyr flare activity cut-off age. Haywood further finds that those stars in the metallicity range of interest here have ages between 6 to 8 Gyr. The fact that Proxima is an 
observed slow-rotator, with a spin-period of 83.5 days [30], is suggestive, therefore, of it being an old thin-disk, largely spun-down star nearing the end of its flare-activity epoch.

The results presented in this analysis, at face value, do not greatly alter the habitability zone characteristics that apply to the newly discovered planet Proxima b (Anglada-Escude et al. [31]). They do, however, carry the profound implication that the planet may be several billions of years older than assumed on the basis that Proxima formed in a coeval manner with $\alpha$ Cen AB. Accordingly, the planetary interior models and the status of any atmosphere associated with Proxima b (see, e.g., $[32,33])$ will need modification, with account being taken of a decidedly non-solar composition and a possible system age of up to 7 or 8 Gyr. Additionally, there would be no reason to suppose that Proxima has had any influence upon the growth of (potential) planets around either $\alpha$ Cen A or $\alpha$ Cen B.

\section{Conclusion}

It has been argued in this paper that the uncertainties associated with the (present day) observational values for the mass, radius, temperature and luminosity of Proxima Centauri are sufficiently well constrained that the Vogt-Russell theorem, as associated with the equations of stellar structure, can be invoked as a means of estimating its metallicity. For the theoretical evolutionary models and the observations to be brought into any reasonable agreement, we find that Proxima must have a significantly lower heavy-element abundance (metallicity) than that derived for either $\alpha$ Cen A or $\alpha$ Cen B. From this we infer that Proxima did not form at the same time or in the same location of the galaxy as the $\alpha$ Cen AB binary.

It is, we would suggest, too early to fully decide the issue of Proxima's status as alpha-Centauri C. While the recent analysis by Kervella, Thevenin \& Lovis [7] begins to make a gravitationally bound status seem more likely, such a result would then present us with a distinct conundrum concerning what are essentially straightforward evolutionary model calculations. Indeed, we find, that the deduced mass, radius, temperature, luminosity, and age are not consistently explained with respect to the theoretical evolutionary models when Proxima Centauri has the same chemical abundance as $\alpha$ Cen $\mathrm{AB}$. The resolution of this problem lies within the continued improvement of the techniques of observational analysis (e.g., radius, luminosity and temperature determinations), the revision of in-put parameters to stellar evolutionary models of very low mass stars (e.g., detailed atmospheric conditions - including molecular opacity and magnetic field effects), and/or, as is most likely the case, in both of these arenas. If the inconsistency between the numerical models (assuming a composition similar to that deduced for $\alpha$ Cen A or $\alpha$ Cen B) and the observations continues to hold, however, then we can only conclude that Proxima Centauri is not alpha-Centauri C. Indeed, we find that while Proxima Centauri may well be our closest stellar neighbour at the present epoch, it still presents us with many challenges that have yet to be clearly resolved.

\section{Acknowledgements}

A great debt of thanks is offered to Richard Townsend and the Ez-Web consortium for making their stellar evolution code publicly available, and for their excellent, user friendly, web-based interface to go with it.

\section{References}

[1] Beech, M. 2012. A journey through time and space: Alpha Centauri. Astron. Geophys. 53, 6.10.

[2] Walke, D. 1979. Alpha and Proxima Centauri: radial velocities and the bound state. Ap. J. 234, L205.

[3] Mathews, R and Gilmore, G. 1993. Is Proxima really in orbit about Cen A/B? MNRAS. 261, L5.

[4] Wertheimer, J., and Laughlin, G. 1995. Are Proxima and Alpha Centauri gravitationally bound? AJ. 132, 1995.

[5] Beech, M. 2009. Proxima Centauri: a transitional modified Newtonian dynamics controlled orbital candidate? MNRAS. 399, L21.

[6] Beech, M. 2011. The orbit of Proxima Centauri: a MOND versus standard Newtonian distinction. Astrophys. Space Sci. $333,419$.

[7] Kervella, P., Thevenin, F., \& Lovis. C. 2016. Proxima's orbit around Alpha Centauri. arXiv:1611.03495v3.

[8] Eggenberger, P. et al. 2004. Analysis of including seismic constraints. $A \& A .417,235$.

Centauri $\mathrm{AB}$

[9] Bazot, M., et al. 2012. A Baysian approach to the modelling of Cen A. MNRAS, 427, 1847.

[10] Beech, M. 2011. Exploring alpha Centauri: from planets, to a cometary cloud, and impact flares from Proxima. The Observatory, 131, 212.

[11] Demory, B. et al. 2009. Mass-radius relation of low and very low-mass stars revisited with the VLTI. $A \& A$. 505, 205.

[12] Mann, A. W. et al. 2015. How to constrain your M Dwarf: measuring effective temperature, bolometric luminosity, mass and radius. Ap. J. 804, 64.

[13] Tayler, R. 1978. In, The Stars: their structure and evolution. Wykeham Publications, London.

[14] Hansen, C., and Kawaler, S. 1994. In, Stellar Interiors: physical principles, structure and evolution. Springer-Verlag, New York.

[15] Segransan, D. et al. 2003. First radius measurements of very low mass stars with the VLTI. $A \& A$. 397, L57.

[16] Doyle, J., and Butler, C. 1990. Optical and infrared photometry of dwarf $\mathrm{M}$ and $\mathrm{K}$ stars. $A \& A .235,335$.

[17] Townsend, R. 2015: http://www.astro.wisc.edu/ townsend/static.php?ref=ez-web.

[18] Paxton, B. et al. 2015. Modules for experiments in stellar astrophysics (MESA): binaries, pulsations and explosions. $A p$. J. Suppl. Ser. 220, article id. 15. 
[19] Eggleton, P. P. 1971. The evolution of low mass stars. MNRAS. 151,351 .

[20] Schlaufman, K., Laughlin, G. 2010. A physically-motivated photometric calibration of $\mathrm{M}$ dwarf metallicity. $A \& A, 519$, id. A105.

[21] Brocato, E., Cassisi, S., \& and Castellani, V. 1998. Stellar models for very low-mass main-sequence stars - the role of model atmospheres. MNRAS. 295, 711.

[22] Cassisi, S. 2011. Very low-mass stars: structural and evolutionary properties. arXiv:1111.6464.

[23] Baraffe, I. et al. 2015. New evolutionary models for pre-main sequence and main sequence low-mass stars down to the hydrogen-burning limit. $A \&$ A. 577, id. A42.

[24] Baraffe, I. et al. 1997. Evolutionary models for metal-poor low-mass stars: lower main sequence of globular clusters and halo field stars. $A \& A .327,1054$.

[25] Haywood, M. 2001. A revision of the solar neighbourhood metallicity distribution. MNRAS. 325, 1375.

[26] Shapley, H. 1951. Proxima Centauri as a flare star. PNAS. 37, 15.
[27] Davenport, J. et al. 2016. MOST observations of our nearest neighbour: flares on Proxima Centauri. Ap. J. 829. article id. L31.

[28] Yadav, R. et al. 2016. Magnetic cycles in a dynamo simulation of the fully convective M-star Proxima Centauri. Ap. J. 833, article id. L28.

[29] West, A. et al. 2008. Constraining the age-activity relation for cool stars: The Sloan Digital Sky Survey Data Release 5 low-mass star spectroscopic sample. $A J .135,785$.

[30] Benedict, G. et al. 1998. Photometry of Proxima Centauri and Barnard's stars using Hubble Space Telescope Fine Guidance Sensor: A search for periodic variations. AJ. 116, 429.

[31] Anglada-Escude, G. et al. 2016. A terrestrial planet candidate in a temperate orbit around Proxima Centauri. Nature, 536, 437.

[32] Luger, R., and Barnes, R. 2014. Extreme water loss and abiotic $\mathrm{O}_{2}$ build-up on planets throughout the habitability zone of $\mathrm{M}$ Dwarfs. Astrobiology, 15, 119.

[33] Brugger, B. et al. 2016. Possible internal structures and compositions of Proxima Centauri b. Ap. J. Lett. 831, article id. L16. 